\title{
AL INTERIOR DE CUARTO APARTE: PLATAFORMA AUTÓNOMA DE ARTE Y CULTURA
}

\author{
Fernando Falconí (FALCO)
}

Fecha de recepción: 08/2016

Fecha de aceptación: 10/2016

\begin{abstract}
Resumen
Este texto habla sobre "Cuarto Aparte: Plataforma Autónoma de Arte y Cultura" activada desde el 2009 como una propuesta colectiva y colaborativa, alterna a la Bienal Internacional de Cuenca, Ecuador. Desde ese año hasta la fecha, articulándonos y articulando individualidades y colectivos de las múltiples escenas locales y nacionales, buscamos visibilizar, reflexionar y debatir sobre obras, proyectos y agendas más allá de los eventos y escenas institucionales, dentro de un ecosistema cultural, artístico, político y social del cual tanto autónomos, colectivos, codependientes e instituciones formamos parte y nos afectamos y relacionamos de diferentes y diversas maneras. Desde el lugar de coordinador general de la plataforma hago una revisión cronológica de la misma, incluyendo su desarrollo de contenidos mediante Franjas así como sus principios y políticas. Reflexiono también sobre la noción de autonomía y procesos de autoconvocatoria, autogestión y autosustentabilidad en el arte y la cultura, con enfoque en el contexto nacional.
\end{abstract}

Palabras clave: Cuarto Aparte, Bienal de Cuenca, Arte, Autonomía, Autogestión.

\begin{abstract}
This text is about "Cuarto Aparte: Plataforma autónoma de arte y cultura" which, since 2009 is a collective and collaborative project, alternating with the Bienal Internacional de Cuenca, Ecuador. Since 2009 to date, individuals and groups articulate multiple local and national scenes in order to show, and debate on works beyond institutional events, within artistic and political ideas which autonomous artists, collective groups and institutions take part and relate to each other in different ways. As the platform coordinator, I make a chronological review, including its content and development as well as its principles and guidelines. I consider the concept of "autonomy" as well as procedures of congregation, auto-management and self-sustainability in art and culture, with a focus on Ecuadorian context.
\end{abstract}

Key words: Cuarto Aparte, Bienal de Cuenca, art, autonomy, self development.

\begin{abstract}
Autor:
Artista transdisciplinar, Master en Arte y Nuevas Tecnologías en la Universidad Europea de Madrid. Licenciado en Artes Visuales en la Universidad de Cuenca, Ecuador. Tiene reconocimientos y selecciones nacionales e internacionales en arte contemporáneo (artes visuales, arte de acción, arte público, inserción de arte en la esfera pública, arte contextual, arte relacional). Fue Coordinador la Carrera de Artes Contemporáneas de la Universidad San Francisco de Quito, donde actualmente es docente. Coordinador general de la plataforma autónoma de arte Cuarto Aparte.
\end{abstract}




\section{ANTECEDENTES}

En el 2007, paralelamente a la IX Bienal de Cuenca, por iniciativa y coordinación de ARTLAB_Creación Contemporánea se realizó la primera edición del formato "Apropósitode" La propuesta "A propósito de la Bienal de Cuenca"2, mediante una plataforma de gestión artística y cultural, convocó, produjo, visibilizó y documentó en la ciudad de Cuenca y a través de Internet una serie de propuestas no oficiales a la Bienal, tanto prácticas (obra física, acciones, instalaciones, proyecciones, etc.) como teóricas (textos), durante los meses de abril, mayo y junio de ese año. Ese evento, además de visibilizar y brindar apoyo autogestionado (sin ningún tipo de dependencia ni compromiso con la Bienal de Cuenca o institución alguna) a los artistas y creadores nacionales e internacionales que participaron en él, dentro de su formato replicable buscaba ser una instancia de reflexión, diálogo y debate en permanente construcción colectiva respecto a los criterios y mecanismos institucionales, curatoriales, municipales y burocráticos relacionados con la escena y los circuitos artísticos y culturales, esencialmente locales y nacionales. Algunas obras a destacar que participaron en este edición de "Apropósitode" fueron: "Cubo Global”, de Italo García (Cuenca), intervención en el Parque Calderón, y "Emerging Art Stars Cuenca, Primer reality show de las artes plásticas", de Michael López Murillo (Colombia). También

1 La web de "Apropósitode", en su edición respecto a la IX Bienal de Cuenca, 2007, estuvo en línea hasta el 2008. Se pueden encontrar ahora registros de ese sitio mediante: https://archive.org/web/

2 "A propósito de la Bienal de Cuenca" fue una coproducción entre ARTLAB_Creación Contemporánea (Falco), Nukanchik People, Arte y Audiovisuales (Juanpablo Ordóńez y Melina Wazhima) y CUCO, Cultura Contemporánea (Hernán Pacurucu y Adrián Pacurucu). el mismo ARTLAB generó la propuesta para la esfera pública "Arte no es censura" (inspirada en el gesto "Arte no es pintura" hecho por Artefactoría en la primera Bienal), a propósito de la censura acontecida en la Bienal del 2007 contra la obra Eric y yo dormidos de Santiago Reyes.

\section{CUARTO APARTE}

\section{CA1.0 - 2009}

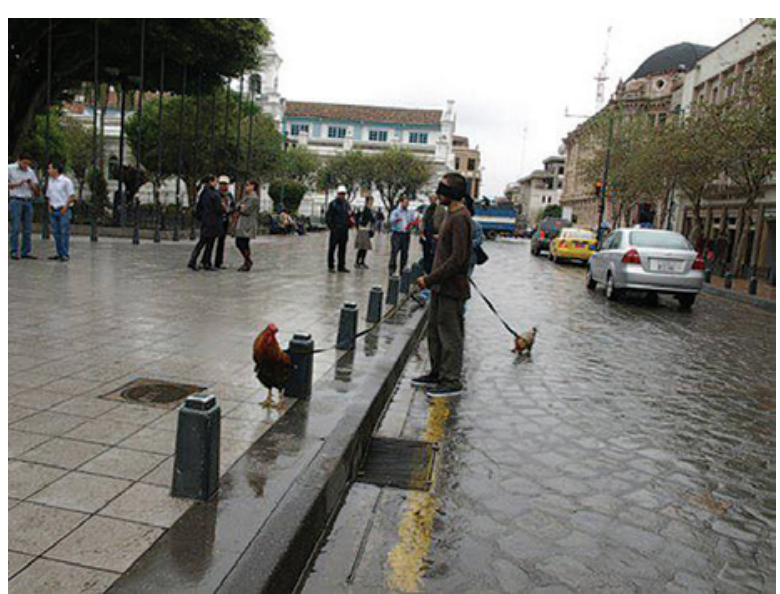

Fig. 1, Franja Ciudad Abierta. "Cuento, fábula y discurso", Performance de Aníbal Alcívar, Parque Calderón, CA1.0, 2009. Fuente: Archivo CA

Tras la experiencia de "Apropósitode" del 2007, algunos artistas y gestores culturales nos mantuvimos en diálogo para continuar en la articulación de una plataforma paralela a la Bienal de Cuenca, autónoma frente a la misma. En el 2009 el amigo, artista y productor Fabiano Kueva ${ }^{3}$ propone un evento que además comprenda una plataforma festiva: espacios de celebración y encuentro distintos a los que tradicionalmente habían manejado las ediciones de la Bienal, de carácter más formal e incluso

3 Respecto a esta articulación colectiva, Kueva apunta: "Cuarto Aparte se reconoce como un nodo más en la trama de gestos/acciones/iniciativas/eventos no oficiales que han planteado preguntas, generado tensiones y abierto horizontes sobre los modos de producción artísticos y culturales en el Ecuador en las dos últimas décadas." 
algunos de acceso restringido o corte elitista. Se integró este propósito al tejido que fuimos elaborando de manera colectiva con diferentes propuestas que surgieron desde lo que se conformaría entonces como un núcleo de gestión y producción Cuarto Aparte, propuestas que involucraban exposiciones, mesas de diálogo, talleres, etc

Es así que se da una nueva "conjunción posible de artistas, colectivos artísticos y productores culturales de Cuenca, pero también de Quito, Guayaquil, Ambato y otras ciudades del mundo, que autoconvocados desde necesidades, empatías y afectos, y autogestionados en diálogo, coparticipación y solidaridad, generamos una plataforma fronteriza/alterna/convergente que desde ese año tiene lugar durante la Bienal Internacional de Arte de Cuenca" ${ }^{4}$, sin intención o pretensión de disputarla, menos aún de ser una Contra o Anti Bienal.

Cuarto Aparte (CA) tuvo su primera versión alterna a la X Bienal en el 2009, con una programación intensiva que incluyó no sólo muestras artísticas sino también proyecciones de videoarte expandido, presentación de publicaciones, intervenciones en espacio público y conciertos de música experimental, logrando importantes resultados a nivel artístico y proponiendo prácticas, debates y agendas que la oficialidad no considera. Tras esta exitosa experiencia y arranque, Cuarto Aparte se posicionó como una plataforma abierta, emergente y plural con la intención y proyección de ser replicada cada dos años. ${ }^{5}$

4 De la declaración de intenciones de Cuarto Aparte:

http://www.cuarto-aparte.blogspot.com/p/cuartoaparte-2011.html

5 El equipo de gestión y producción 2009 (CA1.0) fuimos: CU. Gallery, Nukanchik People, SONO Estudio, LOOPXUS y quien escribe, de Cuenca; Centro
CA2.0 ${ }^{6}-2011$

Esta edición creció en términos de oferta artística, producción, audiencia y visibilidad no solo a nivel nacional, sino internacional. Se diseñaron siete segmentos de programación (a los que llamaríamos Franjas) en varias sedes, con propuestas artísticas de Ecuador, Perú, Brasil, Colombia, Venezuela, Centroamérica y EE.UU.

\section{CA3.0 ${ }^{7}-2014$}

Ese año, Cuarto Aparte mantuvo sus Franjas habituales y además generó el espacio "Casa Aparte", laboratorio creativo y de convivencia para estudiantes de arte y artistas jóvenes de todo el Ecuador. Además, CA se amplió en cuanto a las temáticas y los campos a tratar, por lo que llegó a más público y sectores no sólo artísticos y culturales, sino sociales y comunitarios en la esfera de la sociedad civil y del activismo.

\section{CA4.0 - 2016}

Al momento que escribo este texto hemos avanzado en la organización y programación de la cuarta edición de Cuarto Aparte (CA4.0), a realizarse del 17 al 27 de noviembre de este año. Para optimizar nuestros recursos humanos y logísticos, decidimos reducir el número de

Experimental Oído Salvaje de Quito y Espacio Vacío de Guayaquil. Cabe destacar la colaboración y el uso de las instalaciones del Prohibido Centro Cultural, espacio icónico en el país en donde se realizó la mayoría de nuestras actividades.

6 Gestamos y producimos esta edición: CU. Gallery, Centro Experimental Oído Salvaje, Nukanchik People, SONO Estudio, Taller de Arte Visual 16-63, LOOPXUS, Rocksie Brown y Falco.

7 CA3.0 fue gestado y producido por: CU. Gallery/ Salida de Emergencia, Centro Experimental Oído Salvaje, Nukanchik People, SONO Estudio, Taller de Arte Visual 16-63, LOOPXUS, Lakomuna, Espacio Vacío (GYE), Gabriela Paredes, Cristina Carrasco y Falco. Colaboró en Casa Aparte Juan Montelpare. 
Franjas a producir y delegar producciones específicas, por ejemplo, el Encuentro Nacional de Estudiantes de Arte (ENEA) trabajado en colaboración directa con la "No Aso" Escuela de Artes de la Universidad de Cuenca. Además, se han sumado a la organización colectivos e individualidades relacionados con el arte, la cultura y la gestión cultural. ${ }^{8}$

\section{FRANJAS}

Desde nuestra primera edición CA nos hemos organizado en diferentes segmentos o franjas que contienen nuestra oferta artística y cultural. Del 2009 al 2016 hemos alternado entre las siguientes:

Exposiciones: Muestras artísticas en espacios cerrados, algunas bajo el concepto y curaduría de CA y otras acogiendo propuestas diversas de artistas o colectivos que han solicitado exponer en el marco de nuestra plataforma y hemos revisado la pertinencia y calidad de sus propuestas.

La Sobremesa: Intercambio de ideas y debates en torno al arte y la cultura dentro del formato de un almuerzo con los exponentes y el público invitado. De manera más horizontal y tras la comida, son discutidos estos temas.

Portátiles: Instancias articuladas como mesas de exposición y diálogo en torno a proyectos, procesos, plataformas

8 Contamos por ejemplo dentro del grupo base de gestión al colectivo Lakomuna y la "No Aso" de la Escuela de Artes de la Universidad de Cuenca. Nos mantenemos en CA4.0: CU. Gallery/Salida de Emergencia, SONO Estudio, Blasco Moscoso, Cristina Carrasco, Gabriela Paredes y quien escribe, más colaboraciones de Carli Tacuri, Raúl Armijos, Daniel Coka, Julieta Vidal, Gabriela Vásquez y Ana Vela. y agenciamientos locales, nacionales e internacionales.

Nodos: Diálogos críticos sobre temas actuales, involucrando a diversos agentes sociales, políticos y culturales mas allá de los circuitos artísticos, en diferentes sedes, para ampliar el debate/la acción, y fortalecer/crear redes interdisciplinarias y de campos.

Casa Aparte: Espacio de residencia/ convivencia creativa para estudiantes de arte, artistas jóvenes y emergentes, que comprende un cuarto de proyectos y la organización desde el grupo residente de un tema para la Sobremesa. Incluye también la visualización comentada de portafolios.

Lanzamientos: Presentación de publicaciones actuales de arte y cultura, más diálogo abierto con sus autores y editores.

Talleres/Laboratorios: Espacio para intercambio de saberes y prácticas diversas, ambiente de taller y experimentación para artistas,estudiantesylacomunidadengeneral.

Código Abierto: Zona de acceso y consulta a material textual, gráfico y audiovisual, mas archivos para descargar/compartir. Sala de lectura de publicaciones de diferentes países. Espacio de exhibición/venta de publicaciones de los Lanzamientos.

Ciudad abierta: Serie de intervenciones en espacios públicos de Cuenca basada en una selección de las experiencias desarrolladas en los últimos años, además de una convocatoria para 


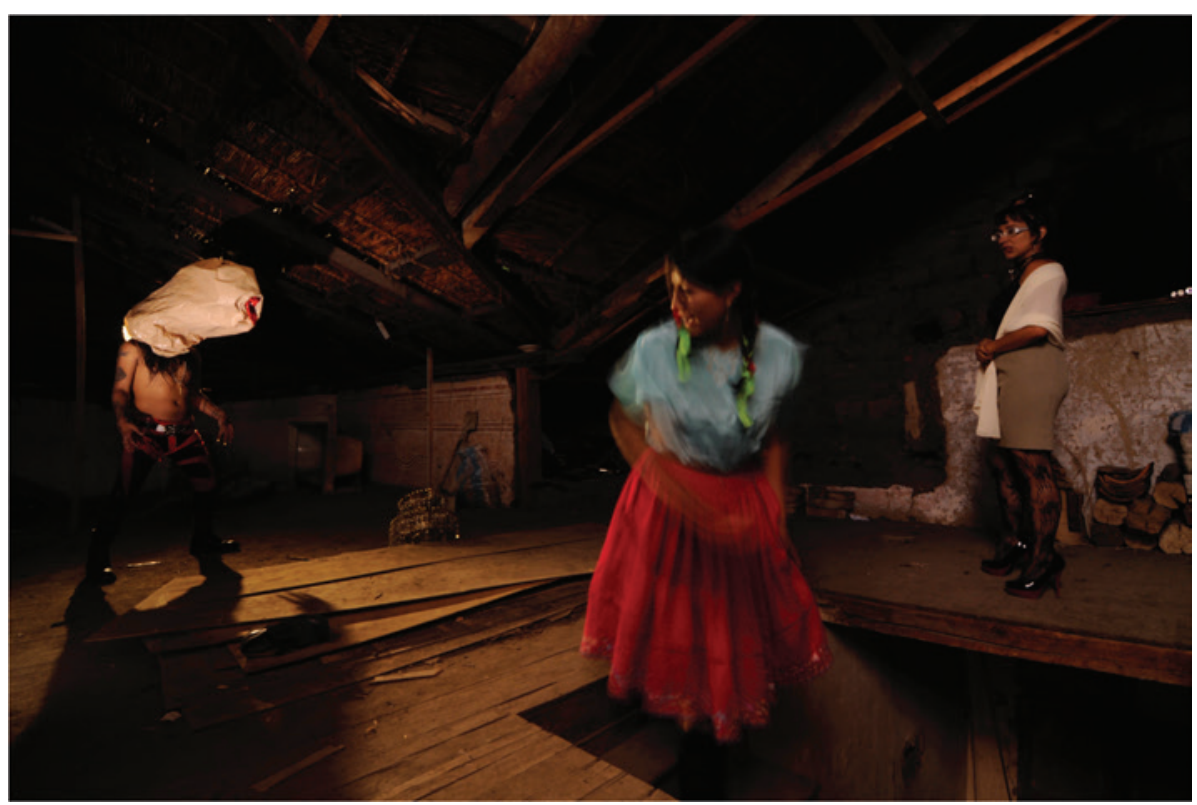

Fig. 3. Franja Laboratorio. Do It Your Self, Taller de fotografía expandida con Rodrigo Etem. Fuente: Archivo CA

una feria de publicaciones.

Proyectables: Selección y proyección de video arte y video experimental en el espacio público (diferentes paredes en la ciudad).

Cuarto Aparte FM 88.1: RADIO LIBRE, señal de radio de Cuarto Aparte. En el 2014, Oído Salvaje trasmitió en vivo $y$ diferido segmentos sobre actividades, diálogos, entrevistas, músicas experimentales y novedades de CA.

Satélites: Presentaciones, diálogos $\mathrm{y}$ activaciones de proyectos e iniciativas desde diferentes modelos de gestión y producción en el Ecuador.

Convergentes: Agendamiento en nuestra programación de propuestas artísticas/ culturales autónomas, exhibidas o desarrolladas en Cuenca en fechas simultáneas a Cuarto Aparte.

Plataforma Festiva: Serie de conciertos y sesiones de DJ y VIJING de diversos estilos musicales y experimentaciones sonoras. Fiestas, shows y performances.

\section{PRINCIPIOS Y POLÍTICAS}

Cuarto Aparte es una plataforma autónoma de arte y cultura, pero es también una red local, nacional e internacional de artistas, colectivos artísticos, gestores culturales y emprendimientos más allá de lo que la institucionalidad asume, legitima y visibiliza como arte y cultura, y la relación de estos con la sociedad. CA se ha basado y sostenido en una economía otra, no determinada por lo monetario: una economía participativa, colaborativa y solidaria con y desde agentes, productores y realizadores activos y propositivos 
del medio, una economía sin fines de lucro, no centrada en el beneficio personal sino colectivo; una economía también de confianzas, afectos, apuestas, voluntades y capacidades.

Frente al aparato institucional, estatal y burocrático, frente a las limitaciones de fondos y subvenciones para la cultura, esta plataforma, red y economía democrática y simbólica plantea modos, sistemas y metodologías otras de pensar, hacer, compartir y relacionarse, desde miradas, posiciones y acciones críticas, sociales y políticas en el arte y la cultura local y nacional, así como desde articulaciones y agenciamientos proactivos y con capacidad de adaptabilidad.

Algo que considero importante es que desde el principio hemos trabajado en CA gente de bases: una diversidad de artistas, actores / productores / gestores culturales, curadores, académicos, docentes, diseñadores, comunicadores, etc. Por un lado, ya manejamos criterios, saberes, haceres, experticias y trayectorias relacionadas con camposy disciplinas específicas, y por otro, durante todo el año estamos en continuo contacto y relación con la producción y gestión cultural nacional, ya sea desde nuestro trabajo directo o desde la relación con nuestros pares, los públicos y los diferentes contextos de las prácticas artísticas, de tal manera que CA toma distancia de ser sólo un evento bienal, una institución cerrada, y es más bien una articulación tanto de bases intergeneracionales, intercontextuales e interdisciplinarias, como una minga de individualidades y colectivos que da cuenta a fondo de toda una trama de escenas y pulsares artísticos en el Ecuador, más allá de las corrientes y legitimaciones dominantes a nivel institucional, burocrático y comercial, y de sus estructuras de poder y relación entre productores y consumidores, incluyendo al mercado del arte y al público en general. En ese sentido, hemos acogido siempre la diversidad y diferencia de criterios y miradas artísticas, estéticas, políticas y de construcción de sentidos tanto individuales como colectivos. En cada edición hemos contemplado además sensibilizar a la sociedad en general (no sólo al sector artístico y cultural) sobre temas relacionados con los derechos humanos, los derechos culturales, los derechos de la naturaleza y la equidad sexogenérica.

\section{AUTOSUSTENTABILIDAD}

Cabría anotar también que CA hasta ahora no ha recibido ningún auspicio económico ni del Estado, ni de las Municipalidades, ni de institución cultural alguna. En las cuatro ediciones que llevamos produciendo esta plataforma, prácticamente todas han sido autofinanciadas. Ahora, la minga, la solidaridad, la creatividad y la afectividad han sido nuestros mayores capitales invertidos, mismos que nos han permitido sacar nuestra plataforma en cada nueva edición, incluso en este período en particular que vivimos una grave crisis en el sistema cultural institucional en específico y en la economía del país en general.

"De la adversidad vivimos", la sonada frase del reconocido artista brasilero Hélio Oiticica da cuenta no sólo de las limitaciones en cuanto al impacto y reconocimiento social del sector artístico y cultural, sino de las limitaciones económicas y de inversión para sus proyectos y emprendimientos, tanto desde el sector público como desde el privado.

9 Esta frase se utilizó como título del primer Encuentro Iberoamericano sobre Arte, Trabajo y Economía (EIATE), desarrollado en Quito en el 2011. El mismo es realizado y convocado por Espacio Arte Actual FLACSO. Se autodefinen como una plataforma para la reflexión crítica sobre las relaciones entre arte y economía. 
Constante y tradicionalmente el arte y la cultura en países como el nuestro se han encontrado en crisis de fondos, de apoyos, de planificación y de programas sostenidos para su desarrollo; claro que la situación actual se ha encrudecido, pero no es sólo de ahora y de estos tiempos críticos para el Ecuador que vivimos esto. Pero ante la adversidad y las crisis, aflora aún más el emprendimiento y la resiliencia, así como el capital humano, creativo y solidario. Desde el principio hemos generado y articulado diversas estrategias para sostener nuestra plataforma. Por ejemplo, desde la primera edición CA creamos una red de espacios propios para recibir a gente que llega de otras ciudades a participar en nuestras actividades: nuestra mismas casas o apartamentos, espacios de familiares o amigos, incluso talleres o lugares alternos. Sobre esto, para las exposiciones artísticas que hemos manejado, también hemos gestionado espacios diversos más allá de "nuestros" espacios (que hasta ahora han sido SONO, Salida de Emergencia/Cu Gallery y la casa de Lakomuna). Hemos hecho actividades en espacios culturales de amigos, como el Prohibido Centro Cultural o la casa Fishbon del Sur; mas, como siempre ,nos han desbordado las propuestas recibidas de exposiciones o actividades culturales y artísticas, hemos utilizado también bares, restaurantes, tiendas, talleres y casas de vivienda, lo que ha generado dinámicas e interacciones otras, más allá de las habituales dentro de la tradición del Museo y el Cubo Blanco. Esto, más toda una Franja que hemos manejado de actividades en el espacio público, ha hecho que hayamos ampliado nuestra relación, incidencia e impacto en y con la sociedad civil en general, generando zonas de intercambio y diálogo de contenidos sensibles, estéticos, reflexivos, poéticos y simbólicos más allá de los sistemas tradicionales, institucionales y muchas veces elitistas relativos al arte en general y al contemporáneo en particular.

\section{AUTONOMÍA}

Como ya anoté anteriormente, CA desde su primera edición no ha buscado ni disputar el lugar y la importancia de la Bienal de Cuenca (además, eso sería imposible dados los presupuestos económicos, de recursos y logísticos que la Bienal maneja y los que nosotros manejamos), ni tampoco ser o asumirnos como una Contra o Antibienal. Recalco también que no nos enunciamos como plataforma independiente, sino como autónoma. Primero, porque autónomos somos ante institución alguna, privada o pública. Somos autónomos en la generación de nuestros sistemas, agendas y contenidos. Somos autónomos ante dependencia ideológica, política, clientelar o económica alguna. Y también somos autónomos porque cuestionamos el concepto y nominación de independientes. Hasta qué punto y retomando un planteamiento de Curatoría Forense ${ }^{10}$ podemos hablar de ser o presentarnos como independientes, cito: "porque la independencia encierra en su enunciación dominación y su correlativa emancipación; y nosotros no creemos en autoridad alguna de la cual liberarnos. Pero además (y en relación al trabajo colectivo y colaborativo), creemos en la co-dependencia como un sistema de trabajo, de amistad y de deseo." ${ }^{11}$

10 "Curatoría Forense es un grupo multidisciplinario de trabajo dedicado al arte contemporáneo en Latinoamérica, que en el tiempo del texto citado era coordinado por Jorge Sepúlveda T., crítico y curador, Ilze Petroni Ph.D., investigadora de arte contemporáneo, Judith Le Roux y Karina Quinteros, Artistas Visuales.”

11 Autónomos, no independientes, Curatoria Forense, http://www.curatoriaforense.net/niued/?p=1215 
"En ese sentido y dentro de estructuras hegemónicas, se plantea y asume la figura de autónomos (no subordinados) antes que independientes, ya que de todas maneras artistas, críticos, gestores culturales, instituciones, entes académicos, municipales y estatales, estamos interrelacionados dentro de un sistema de campos (como los planteados por Pierre Bourdieu) (2002), en este caso el campo artístico y cultural, en que de una manera más o menos dirigida, conciente o explícita, todos nos estamos interrelacionado e influyendo mutuamente en distintos grados, más que como una dispersión o una generación aislada de instituciones, plataformas y escenas, como una ecología sociocultural, económica, estética, simbólica y relacional." 12

De esta manera, asíes comovemos nuestra relación con la Bienal (y con la institucionalidad artística en general): ambos somos parte de un ecosistema en el que la diversidad, la diferencia e incluso las tensiones, los debates y los desacuerdos no sólo que son buenos, sino necesarios dentro de la complejidad de dicho ecosistema del cual todos formamos parte, incluso pudiendo enriquecer y enriquecernos mutuamente dentro de las diversos modos de participación y afectación en el mismo. ${ }^{13}$

12 Párrafos de mi ensayo: "Nodos y modos críticos y colaborativos. Apuntes sobre gestos, escenas y escenarios en la producción artística ecuatoriana”. Pensar el arte. Actas del Coloquio sobre arte contemporáneo en Ecuador, Universidad de Cuenca, 2014.

13 De nuestra parte, siempre hemos estado abiertos a tener espacios de encuentro y diálogo con la Bienal, en el sentido de poder intercambiar, compartir y debatir visiones, conocimientos y experiencias convergentes o divergentes, pero de parte de esa institución nunca ha habido el real interés o invitación a un encuentro. Recuerdo que en el 2014 invitamos a la directora de la Bienal de ese entonces a compartir un espacio de opinión con nosotros en la radio, pero no aceptó. Repetiremos la invitación al director de la XIII Bienal.
Algo muy alentador a nivel personal (y para el núcleo fundador de Cuarto Aparte), por ejemplo, es constatar como nuevas generaciones de artistas y gestores de todo el país están tomando la posta de esta plataforma, asumiendo compromisos, responsabilidades y trabajos comunitarios y en comunidad, con liderazgos incluidos. Esto, igualmente, no sólo que es bueno y enriquecedor para todos, sino es sano, justo y necesario. Finalmente, asumimos a CA no sólo como una práctica cultural, artística y de gestión, sino como un ejercicio político y una toma de posición ya no sólo desde el discurso, el análisis y la crítica, sino desde la propuesta, el hacer y el compartir. Desde las limitaciones económicas, el vértigo y el riesgo, pero también desde la celebración de las sinergias, las confianzas y los afectos. Y desde la firme certeza y satisfacción de estar aportando colectiva y sostenidamente más allá de la oficialidad y la institucionalidad artística en el arte y la cultura ecuatoriana.

\section{Bibliografía}

Abad Vidal, J.(ed.): Pensar el arte. Actas del Coloquio sobre arte contemporáneo en Ecuador. Cuenca (Ecuador), Universidad de Cuenca, 2014.

Cuarto Aparte (22 de agosto de 2016). Declaración de intenciones de Cuarto Aparte. Recuperado de http://www.cuarto-aparte. blogspot.com/p/cuarto-aparte-2011.html

Bourdieu, P. (2002). Las Reglas del Arte: Génesis y Estructura del Campo Literario. Barcelona: Anagrama.

Falconí, F. "Apropósitode". A propósito de la Bienal de Cuenca, 2007. [Fecha de consulta, agosto 2016]. Disponible en: ht tps : / / w e b.archive.org/ 
web/20080414161249/http://www. apropositode.com/apropositode2/index.php

Curatoria Forense (22 de agosto de 2016). "Autónomos, no independientes" Recuperado de http://www.curatoriaforense. net/niued/?p=1215 